\title{
A Subjective Appraisal of Aseptic and Sterilizing Practices in General Dentistry
}

Louis Touyz ZG*

Department of Dentistry, Canada

Keywords: Antisepsis; Infection; Microbiota; Practitioner

ISSN: 2578-0042

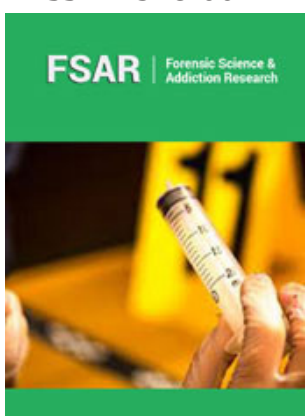

*Corresponding author: Louis Touyz ZG, Department of Dentistry, Canada

Submission: 眥 April 03, 2019

Published: 此April 30, 2019

Volume 4 - Issue 4

How to cite this article: Louis $\mathrm{T} Z$ A Subjective Appraisal of Aseptic and Sterilizing Practices in General Dentistry. Forensic Sci Add Res. 4(4). FSAR.000598.2019.

DOI: 10.31031/FSAR.2019.04.000597

Copyright@ Louis Touyz ZG, This article is distributed under the terms of the Creative Commons Attribution 4.0 International License, which permits unrestricted use and redistribution provided that the original author and source are credited.

\section{Background}

Few current 2019, general dental practitioners recall those days when antisepsis was relatively primitive compared to today. There was a time up to the mid twentieth century 1900 to 1960. When soap and hot water were considered adequate for asepsis (reducing viable organic organisms to the level of no transmission of infection). Washing hands and scrubbing finger-nails under running tepid tap-water with an antiseptic phenol-laced soap and sing "Happy birthday to oneself twice, was considered adequate to ensure manual cleanliness no transfer of microbes. Most general dental practitioners and oral health care workers like Oral Hygienists, dental assistants and nurses, all practiced with un-gloved hands. Many did not wear face masks. Eye protection was with ordinary spectacles. Exodontics and minor oral surgical procedures were done with naked hands and the likelihood of receiving or transferring any infection was considered negligible. Some endodontists claim they could digitally feel when endo-reamers reached the apex. Alginate impressions were kept moist with gauze soaked in tap-water and transported thus to technicians, with no regards to possibilities of infection. Boiling water "Sterilizers" were kept boiling all day to 'sterilize hand instruments."

Each time one opened the lid covering the boiling cauldron, your spectacles would steam up. Dry heat sterilization was kept for special endodontic instruments (at $120^{\circ} \mathrm{C}$ overnight), and most heat labile supports like rubber tubing and bowls, plastic instruments, mixing spatulas, were simply wiped down with surgical ethanol $(90 \% \mathrm{~V} / \mathrm{V})$. The entire operatory could be wiped down with ethanol or some other antiseptic liquid. In the mid 1950's the contraceptive pill for females was introduced and then globally in the 1960's much libidinous behaviour followed. In the 1970's the scourge of HIV started to manifest when many young homosexual men between 18 and 28 were dying from AIDS. Being infected with HIV, was considered a death sentence as most HIV infected patients succumbed to AIDS with a decade of the initial infection. HIV infection from any and all sources was deemed possible, and a patient (Kimberly Bergalis) accused and her gay Dentist (Dr. Acer) of infecting her with HIV. History proved this accusation to be spurious if not false, but the publicity generated in the seventies from this case shocked the entire dental profession world-wide into adopting more stringent disinfection practices.

Aim

This subjective appraisal recalls anachronistic practices and underlines the necessity to strive for and maintain improved sterilization practices in general dentistry.

\section{Current Practice}

The vast majority of licensed general dental practitioners now used proven techniques and practices to sustain infection control and minimize to the point of extreme, the lowest chances of transmitting or nurturing any infection. These practices are now routine, are taught to aspiring dental students as part of their training, and standards are sustained through periodic assessments and monitoring by professional licensing bodies. Placing an ungloved hand into any orifice now is considered an assault, and dentists now not only wash their 
hands regularly between patients, but also don clean sterile gloves for any examination or intra-oral work to be done. Face masks and eye protection is now behind impenetrable masks and transparent eye screens or spectacles. Most practices have mini-autoclaves that successfully sterilize [leaving no viable organic material] using steam or other gases and/or vapours (like ethylene at $120^{\circ} \mathrm{C}$ under 10 kilos of pressure) for all instruments prior to use. Disposable clothing is often used, or sterilizing laundry practices used for reusable items, like covers, towels, cloth screens and gowns.

Cleaning and sterilizing protocols are now part and parcel of contemporary dental practice. Highly effective disinfectant liquids are freshly prepared daily and used to disinfect all furnishings in the operatory. At night many operatories have ultra-violet light burning to ensure surface disinfection. Even sterile disposable over-gowns, head-coverings and foot covers may be used to assist in sustaining a disinfected environment. Impressions are mixed with antiseptic solutions and transported in disinfectant liquids to protect dental technicians.

\section{Discussion}

Many people think the new approaches are "overkill" and add unnecessarily to the high cost of dentistry. Yet since these measures were introduced it is extremely rare that any infections from dental practitioners have ever been reported. Assuming there are some 50, 000 registered practicing dentists in North America, and that each sees about 10 patients a day for 200 working days a year, the situations for possibly being infected would be 100, 000, 000 each year!!!! That not one case of transmission of infection from registered modern practicing dentists has been noted now for decades speaks volumes to the success of the modern approach to infection control in dental operatories.

\section{Conclusion}

One unnecessary transfer of infection from dentist to patient, or vice versa, with any morbidity or mortality would be one too many. Technology introduces novel ideas regularly to improve the dental profession, but the fundamentals of infection control are prime and must be rigorously sustained for the health of all dentists, their patients and the whole profession. 